\title{
Construcción subjetiva de la experiencia de los adolescentes-jóvenes de secundaria
}

Mtra. Juana Yesenia Cruz Ramírez, Mexicana.

ORCID: https://orcid.org/0000-0001-8602-6874

yeseram9@ hotmail.com

Instituto Superior de Ciencias de la Educación del Estado de México

Recibido: 21 de junio del 2020

Aceptado: 28 de septiembre del 2020

\section{Resumen}

En este artículo se entrelazan referentes epistémicos, teóricos y empíricos que permiten comprender la construcción subjetiva de los adolescentes-jóvenes de secundaria desde un posicionamiento con base en el pensamiento postabismal y la perspectiva configuracionista, ambas propuestas conllevan a repensar al sujeto juvenil como protagonista de su propia constitución subjetiva. En ese sentido, asumo a la investigación como un constructo social que implica una interrelación entre la investigadora con los investigados en un horizonte de igualdad, reciprocidad y alteridad; considerando al sujeto singular, histórico, con experiencia y en constante formación, en una constante reconfiguración de su propia subjetividad. Aquello me posibilitó repensar a la secundaria como un escenario social habitado por los adolescentes-jóvenes cuyas voces dan cuenta de su constitución subjetiva, siendo que su experiencias no se reducen solo a procesos socializadores en un espacio instituido, sino que traspasan esos recorridos al tensar y dar sentido a la escuela como escenario de acción trastocando su vulnerabilidad y movilizándolos en una apropiación a la que se aferran de manera pasional a sentirse vivos; bajo condiciones de desigualdad y violencia ellos son expuesto a experiencias que los hace vibrar y saber de afectos o efectos, los adolescentes-jóvenes en la presente investigación son considerados como sujetos abiertos, sensibles, con ímpetu de una aventurarse a vivir, padecer y ser.

Palabras claves: construcción subjetiva, experiencia, adolescentes-jóvenes. 


\title{
Subjective construction of the experience of high school adolescents-young people
}

\begin{abstract}
In this article, epistemic, theoretical and empirical references are intertwined that allow us to understand the subjective construction of high school adolescents-young people from a position based on post-abyssal thinking and the configurationist perspective, both proposals lead to rethinking the juvenile subject as the protagonist of their own subjective constitution. In this sense, I assume to the investigation as a social construct that implies an interrelation between the researcher and those investigated in a horizon of equality, reciprocity and otherness; considering to singular subject, historical, with experience and in constant formation, in a constant reconfiguration of his own subjectivity. That made it possible for me to rethink high school as a social scenario inhabited by adolescents-young people whose voices give an account of their subjective constitution, as their experiences are not only reduced to socializing processes in an instituted space, but they go beyond those routes by tensing and give meaning to school as a scenario of action by disrupting their vulnerability and mobilizing them to an appropriation to which they passionately cling to feel alive; under conditions of inequality and violence, they are exposed to experiences that makes them vibrate and know about affections or effects, adolescentsyoung people in this investigation are considered as open, sensitive subjects, with the impetus to venture to live, suffer and be.
\end{abstract}

Keywords: subjective construction, experience, adolescents-youth.

\section{Introducción}

El presente artículo se originó como parte de la discusión central de la investigación: "La experiencia de los adolescentes-jóvenes de secundaria en contextos de violencia", realizada como doctorante en el Instituto Superior de Ciencias de la Educación del Estado de México (ISCEEM). El debate giró en torno a la configuración subjetiva de los adolescentes-jóvenes desde la articulación entre lo estructurado y lo subjetivo como referentes constitutivos de su formación y transformación. Con el propósito de comprender los procesos de construcción subjetiva se aludió 
a la horizontalidad mediante un desafío epistémico que tiene como punto relevante y central la dialogicidad en una posibilidad de reconstrucción a través de las narrativas de nuestras experiencias en el intercambio recíproco entre los investigados y la investigadora en la producción de conocimientos.

Para la construcción del artículo se retomó de la investigación mencionada, los posicionamientos epistémicos, teóricos y metodológicos asumidos, a partir de los cuales se abordó el objeto de investigación, así como los conceptos centrales que emergieron desde lo teórico y empírico con la intención de visibilizar el protagonismo de adolescentes-jóvenes como sujetos de conocimiento, experiencia e historicidad, con capacidades de resistir y construir en estrecha relación con el orden y la cultura, configurando horizontes que dan sentido a la constitución de la subjetividad como un modo de mirarnos y situarnos en el mundo desde nuestras diferencias y singularidades.

En ese tenor es importante considerar a la secundaria como el escenario social donde la construcción subjetiva cobra sentido a partir de las relaciones, interacciones de construcción y deconstrucción, un lugar de formación y transformación. Desde ahí comienza a visibilizarse la interrelación inicial entre adolescentes-jóvenes, experiencia, subjetividad y violencia, para entender los procesos de configuración de ellas y ellos en la secundaria.

El escrito conlleva una parte del trabajo de construcción, conocimiento y reflexión frente a la experiencia de constitución subjetiva, recuperando con ello mi implicación frente a un proceso de investigación que interpeló mi propia constitución personal y profesional en la experiencia de repensarme junto a otros en condiciones económicas, sociales y culturales, lo que me permitió un autoconocimiento sobre los modos de estar y ser en un contexto complejo. Y es a través de la epistemología crítica que me planteo el repensar la realidad social como la articulación entre estructura, subjetividad y acción social (De la Garza, 2018).

Para dar contenido a la misma, enuncio algunos aspectos: primero, hago referencia a los sustentos epistémicos y teóricos que construyen la subjetividad de los sujetos desde una horizontalidad como posibilidad de construcción sustentada en el pensamiento post-abismal y el configuracionismo; en un segundo momento, abordo las dimensiones teóricas que reconocen a la 
experiencia en un proceso de reflexividad ${ }^{1}$, diría Larrosa; en donde se manifiesta una trasformación de ida y vuelta en el propio sujeto a partir de un acontecer que cimbra y desplaza sus conocimientos, sentimientos y relaciones con otros. En un tercer momento, de este proceso de construcción emergen voces de adolescentes-jóvenes de secundaria como un proceso generador de conocimientos, una posibilidad de comunicación entre distintos modos de ser y estar en el mundo. Para finalizar, dejo algunos referentes que dan apertura a otras miradas de construcción y transformación.

\section{El pensamiento post-abismal y el configuracionismo}

Con base al pensamiento post-abismal, el conocimiento se construye en un plano de igualdad de reconocimiento, dejando de pensar en lo otro como lo ajeno, lo extranjero, sino dentro de un ámbito de respeto y reciprocidad; parto desde la diversidad de saberes para repensar desde la sociología de las ausencias como una alternativa "para intentar mostrar que lo que no existe es producido activamente como no existente, como una alternativa no creíble, como una alternativa descartable, invisible a la realidad hegemónica del mundo" ( Santos, 2009, p.23), es a partir de conocimientos de lo/ los diferentes en la investigación que surge una necesidad de repensar-nos entre sujetos, reflexionando la relación entre estructura y subjetividad desde la perspectiva configuracionista particularmente con De la Garza (2018), reconociendo en la subjetivación la presencia de una estructuración y la posibilidad de actuar frente a una realidad concreta.

Mirar del otro lado de la línea, aquella que si bien reconoce la configuración del sujeto donde suele mirarse solo el plano estructural, pero no lo estructural en lo subjetivo. El configuracionismo apertura a reconocer esa posibilidad de implicación en el proceso de investigación con los sujetos que investiga, para reconocer e integrar en la investigación el proceso de conocimiento.

\footnotetext{
${ }^{1}$ Para Larrosa (2006), la reflexividad tiene que ver con el me de eso que me pasa. Es la reflexividad en termino de reflejo, de salida y regreso, de ahí que señale que la experiencia es un movimiento de ida y vuelta, "un movimiento de ida porque la experiencia supone un movimiento de exteriorización, de salida de mí mismo, de salida hacia afuera, un movimiento que va al encuentro con eso que pasa, al encuentro con el acontecimiento. Y un movimiento de vuelta porque la experiencia supone que el acontecimiento me afecta a mí, que tiene efectos en mí (p.90)
}

Revista RedCA octubre 2020 - enero 2021 ISSN: 2594-2824 Vol. 3 Núm. 8 
Detenernos a comprender el tiempo, las circunstancias y los lugares en constante movilidad, considerando a la sociedad posmoderna como un modo de estar en el mundo, de imaginarios a través de los cuales los adolescentes-jóvenes construyen su identidad; por ello se hace necesario detenerse a repensar en los modos construidos, considerando los conocimientos que nos han constituido desde lo discursivo y estructural, configurando así la subjetividad. Donde nos diría Santos (2010) el pensamiento ha operado desde líneas abismales de la exclusión, la monocultura, las prácticas deshumanas, negando con ello al otro y a los otros, estructurando el conocimiento moderno desde sus discursos e intereses.

Pensar en la constitución del sujeto en la modernidad conducía a considerar la razón y la libertad como aspectos esenciales para que el hombre llegara a la felicidad, para lo cual necesitaba la ciencia y la técnica para legitimar el deseo por el progreso; es aquí donde tanto la legitimidad como los discursos se relacionan como la necesidad de adaptación a un orden dominante que regula y controla todo acto individual y social. Entonces, la racionalidad científica, la obsesión por el sueño tecnológico y la administración de la libertad, son elementos estructurales para lograr un pensamiento uniformado, eficiente y productivo lo que se incorporaba a la subjetividad del sujeto como una condición natural y social dirigida al desarrollo humano.

Posteriormente, con la mundialización de los capitales y el crecimiento del sistema capitalista con base a la expansión de los mercados, se determinan formas de vida e imaginarios sociales impuestas bajo dicho mandato; sin embargo, no todo es enmarcado a ese orden, existen líneas de fuga que nos alumbran desde el pensamiento postabismal a mirar otras líneas de pensamiento que no reduzcan a la realidad ni al sujeto, sino que reconozcan que la construcción del conocimiento implica generar procesos de producción y valoración de diversos saberes, diversos sujetos, como enunciaría Santos, se apertura "desde el reconocimiento de que la exclusión social en su sentido más amplio adopta diferentes formas según está determinada por la línea abismal o no abismal (2010, p. 31). En el caso de la presente investigación, se reflexiona sobre los adolescentes-jóvenes desde sus distintos espacios, saberes y estéticas que muchas veces son negados por el mundo adulto mediante reglas, prejuicios, estéticas occidentalizadas, legitimados por una élite imponiendo lo aceptable de acuerdo con leyes universales, y donde cada uno de los pensamientos y acciones deben estar supeditadas a ese orden.

Revista RedCA octubre 2020 - enero 2021 ISSN: 2594-2824 Vol. 3 Núm. 8 
Desde esa mirada se acogen los nuevos problemas del mundo globalizado frente a las necesidades de los sujetos por sobrevivir, autoconocerse, repensarse desde lo impensable del pensamiento occidentalizado-abismal, una visión crítica da apertura a la posibilidad de un pensamiento alternativo, una construcción epistémica que no solo visibilice las estructuras de poder y saber, sino que a partir de ese orden social reconstruya el poder y saber desde la construcción ética y política desde los sujetos, diría Santos (2010) de los de abajo, los invisibilizados.

El pensamiento postabismal y el configuracionismos permiten reflexionar la realidad y la construcción del conocimiento desde otros matices para ir comprendiendo la construcción subjetiva de los adolescentes-jóvenes de secundaria en contextos de violencia. De la Garza con el configuracionismo sustentó en el proceso de subjetivación la presencia de lo estructural ${ }^{2}$ y la reconfiguración como una posibilidad frente a una realidad en constante movilidad; esta perspectiva me permitió reconocer al sujeto como creador de la transformación social que hoy se hace urgente para desmantelar los discursos bajo los cuales la subjetividad juvenil se ha soslayado, y por tanto, la violencia que emana del mismo orden; en otro momento, para reflexionar otros modos de reconfiguración mostrados como una transformación que emite ecos de alternativas de reconfiguración subjetiva, la implicación del investigador se hace presente desde lo que pasa y nos pasa, como ese acontecimiento que deja marcas de huellas para continuar caminando por senderos éticos y políticos, reconfigurando a partir de rupturas e interacciones con factores estructurales y posibilitadores que mediante contradicciones y tensiones de poder van entrelazándose en esos procesos constitutivos, entonces "el problema principal no es dar cuenta de lo dado, sino de lo dado-dándose, no como simple devenir, sino como articulación de la voluntad con condiciones dadas-dándose" ( De la Garza, 2018, p.215)

El acercamiento a la comprensión de la experiencia de los adolescentes-jóvenes de secundaria en contextos de violencia analizando las relaciones entre estructuras y subjetividades

${ }^{2}$ Cuando aludo a la estructural me remito a las reglas, instituciones (escuela, familia, iglesia), discursos, jerarquizaciones, objetos, valores, interacciones que acondicionan, presionan, inducen y vigilan el cumplimiento de un código de conducta, que al no acatarse por los sujetos son sancionados, se establece un orden que delimita sus espacios de acción con base a una racionalidad que establece una lógica y un fin.

Revista RedCA octubre 2020 - enero 2021 ISSN: 2594-2824 Vol. 3 Núm. 8 
se abordó desde la noción de configuración con base en el sustento De la Garza (2018) que la explica como una red que permite acceder a códigos subjetivos, estructurales, funcionales:

Con articulaciones de claras a oscuras, permite captar mejor ese dinamismo y analizar las potencialidades de especificación de lo ambiguo a través de prácticas. Es decir del espacio de posibilidades para la acción no puede ser visto como una jaula de hierro (De la Garza, 2018, p. 233)

La racionalidad instrumental bajo las cuales organizamos y pensamos nuestra realidad, y por tanto establecemos nuestras interacciones; bajo esa mirada somos producto de un sistema y nuestra función social es una simple reproducción social de los adultos a los jóvenes, una lectura limitada en el proceso de configuración. Sin embargo, para De la Garza la estructura no se reduce a las relaciones objetivadas, "en esta medida no son puramente físicas sino intersubjetivas, para ser subjetiva tiene que implicar un mínimo de reflexividad, de reconstrucción o verificación de significados" (2018, p.11); el autor considera que existe una multiplicidad de interacciones, las cuales emanan de estructuras pero no se detienen, sino al contrario se continúan movilizando, interactuando y actuando creando sentidos.

Por lo tanto, los jóvenes se encuentran inmersos en estructuras donde la violencia se hace presente en la escuela, la familia y la comunidad; es a través de sus acciones y prácticas que se reconfiguran las formas de actuar e interactuar con esas estructuras, y donde se producen otros modos de ser y estar en la sociedad; a partir de las relaciones entre sujetos frente a múltiples códigos se da sentido a situaciones concretas, generando así una continua e inacabada relación entre estructura, subjetividad y acción.

Entonces, a la subjetividad habría que entenderla como un proceso de dar sentido por parte de los sujetos sociales, posibilitando que entre estructuras objetivadas y acciones la subjetividad sea una mediación necesaria. Para los jóvenes de secundaria, esa configuración subjetiva se hace presente a partir de sus lugares, códigos, interacciones, cultura, pero sobre todo en cómo han vivenciado la violencia en esa mediación necesaria, diría De la Garza.

A partir de las acciones que son parte del sujeto en una relación (conocer, sentir y accionar), es fácil localizar ciertas estructuras y sentidos que permiten a los sujetos tomar decisiones; en otros 
momentos estas acciones están en constante reconfiguración en una realidad caleidoscópica ${ }^{3}$ con componentes simbólicos diversos donde las narrativas con los jóvenes expresan parte de las relaciones entre estructura, subjetivación y acción.

La reconstrucción de las relaciones entre estructuras, subjetividades y acciones, particularmente de las configuraciones subjetivas concretas no pueden ser reducidas a una teoría general, porque la realidad en parte objetiva y en parte subjetiva se reconstruye permanentemente por medio de una multirreferencialidad como una necesidad de diversas lecturas psicológicas, organizacionales, institucionales y sociológicas, todas estas complementarias, completamente heterogéneas las unas con respecto a las otras. Cabe destacar, nos diría Ardoino (1998), que la multirreferencialidad no pretende agotar la riqueza del objeto sino visibilizar distintos puntos de vista con respecto a la realidad social compleja siendo una de sus atribuciones no estar totalmente determinada únicamente por elementos estructurales, sino que en ambos mundos objetivo y subjetivo se vinculan en la construcción del conocimiento y las interacciones sociales como un proceso social que emana sentidos y significados en una realidad de reconfiguración constante que apertura posibilidades diversas de estar.

\section{Experiencia adolescentes-jóvenes de secundaria, aproximaciones conceptuales}

Entender a la experiencia como un proceso de subjetivación ${ }^{4}$ desde una lectura a partir del saber histórico que apertura con base en el texto de la historia de la sexualidad Foucault (1999) como un proceso histórico de correlación en el que se entrelazan la formación de los saberes, los sistemas de poder que regulan su práctica y formas de subjetividad en las que constituyen en su propia interioridad, desde lo que para Foucault sería, "el arte de la existencia".

\footnotetext{
3 "Que no hay acción pura ni metas puras, pero sí predominio mediado de algún tipo", ya que la realidad así como las relaciones sociales desde el posicionamiento explicito por De la Garza señala que cuando hace referencia a lo caleidoscópico como las diversas aristas de algún problema, como económico, político, cultural ( 2018)

${ }^{4}$ Para Foucault analizar "el sujeto" era iniciar a " buscar cuáles son las formas y las modalidades de la relación consigo mismo por las que el individuo se constituye y se reconoce como sujeto (1999, p.9)

${ }^{5}$ Las prácticas sensatas y voluntarias por las que los hombres no sólo se fijan reglas de conductas, sino que buscan transformarse a sí mismos, modificarse en su ser singular y hacer de su vida una obra que presenta ciertos valores estéticos y responde a ciertos criterios de estilo ( Foucault, 1999, p.14)
}

Revista RedCA octubre 2020 - enero 2021 ISSN: 2594-2824 Vol. 3 Núm. 8 
Desde una lectura sociológica de crítica a la modernidad para Touraine (2000), se comprende como una construcción del sujeto de la experiencia que reúne al "hombre interior" de la cultura, al "mundo exterior" de las técnicas y mercados para hacer emerger al sujeto capaz de asociar en una experiencia de vida la racionalidad instrumental y la identidad cultural y psicológica, como una voluntad que da sentido a su vida personal y social.

Ambos autores convergen en la construcción subjetiva del sujeto desde acontecimientos históricos dados (lógica instrumental, sujeción al orden social), en el caso de Foucault y Touraine, a partir de la crítica a la modernidad comienzan a vislumbrar otros horizontes de constitución por parte del mismo sujeto que es capaz de reconstruirse a sí mismo mediante un reconocimiento propio como objeto de conocimiento y sobre las prácticas que permiten transformar su modo de ser.

Y desde una dimensión pedagógica con Larrosa, nos permite acceder a la reflexividad como "un principio de transformación" que apertura el reconocimiento de la interioridad de uno mismo en un devenir plural, creativo y bajo esa enunciación, la experiencia es "eso que me pasa"; se entrelazan las dimensiones enunciadas en el proceso de formación a partir de la exterioridad, subjetividad y pasión, esta última como una acción que nos lleva a desplazarnos a un lugar distinto.

Esta constitución de la experiencia nos hace repensar nuestra propia manera de configuración de lo que me pasa, un antes y un ahora; en ese proceso de construcción subjetiva en el cual nos posicionamos como un sujeto de experiencia interpelada por nuestra historicidad, mi personalidad, y mi sensibilidad.

Cada uno de los anteriores autores han brindado componentes teóricos que permiten en primer instancia, analizar y reflexionar los distintos posicionamientos subjetivos con respecto a la experiencia; en un segundo momento, las interacciones a través de las narrativas constituidas por la experiencia dan muestra de lo que somos, permitiendo reconstruirnos mediante una narrativa de la experiencia de sí, no es algo que se produzca en un soloquio, en un diálogo íntimo del yo consigo mismo (Larrosa, 2018).

Las dimensiones teóricas reconocen a la experiencia como un proceso de reflexividad, diría Larrosa en donde se manifiesta una trasformación de ida y vuelta en el propio sujeto a partir de un 
acontecer que cimbra y desplaza sus conocimientos, sentimientos y relaciones con otros, en acciones consigo mismos y quienes lo rodean.

Para Touraine (2000), la presencia del sujeto en el proceso de subjetivación enuncia la individuación desde la experiencia vivida como una resistencia en el proceso de reconstrucción de su propia identidad con base a una articulación del exterior al interior, como una experiencia de la acción del sujeto consigo mismo y con los otros, como un principio de alteridad en un plano de las diferencias donde construirse es parte constitutiva de la experiencia.

La experiencia estrecha vínculos con lo exterior, lo histórico, lo que acontece, de ese algo que no soy yo en un principio de alteridad-exterioridad, argumentado por Larrosa como "eso que me pasa"; lo que se relaciona con el acontecimiento del qué de la experiencia, en lo concerniente a la investigación, lo que les pasa a los jóvenes de secundaria en contextos de violencia que si bien es algo externo proveniente de un tiempo y espacio de manera dada, no es reducido a una generalidad, sino precisamente pasa en mí en lo que soy, pienso y siento; un principio de subjetividad, en donde el sujeto emerge en el nivel de la experiencia individual y la voluntad de cada uno para dar sentido a su vida (Touraine, 2000), donde la experiencia es siempre singular, donde cada uno la hace o padece de manera irrepetible y pluralista. La singularidad desde su propia experiencia, suya y de nadie más, que brinda sentido desde las huellas o recuentos de un pasaje recorrido, dándose a la vez apertura y movilidad en ese proceso de formación, reflexividad y transformación del propio sujeto en su propia constitución.

Para cada uno de los autores, ser sujeto de la experiencia conlleva a comprender al sujeto como productor en un campo de saber, poder, alteridad, subjetivación y pasión; reconociendo al sujeto, su constitución e interrelaciones con aconteceres y alteridades enmarcadas en tiempos y espacios que posibilita los devenires del proceso formativo presente, donde las relaciones surgen entre sujetos que dicen, callan, cuestionan e indagan; dando apertura a lo pedagógico en la escuela, por ser siempre, diría Contreras “experiencia de la relación y extrañeza del otro" (2010, p.17).

Bajo ese análisis, entendemos que la experiencia como eso que me pasa remite a la subjetividad del sujeto a partir de lo extraño, lo propio y las diversas acciones presentes en lo ajeno, 
lo inaccesible que permite acontecer, reflexionar y transformar-me a partir del reconocimiento de quién soy en mi relación con los otros.

\section{Compartiendo experiencias con los adolescentes-jóvenes de secundaria}

Hacer referencia al sentido de la escuela secundaria es visibilizar a los sujetos que se construyen a partir de lo que son, aprenden y comparten, formándose en las relaciones consigo mismos y con los otros. Hablamos de los protagonistas de las microhistorias que otorgan conocimientos y acciones a la vida cotidiana escolar a partir de sus modos de ser y estar; pensamos en el sujeto históricamente en un tiempo y espacio, como el centro de acción. Como señaló Martínez (2012) el sujeto es potencia, posibilidad, poder constituyente, resistencia, voluntad de acción, solidaridad, pero también es debilidad, egoísmo, poder instituido, función social.

Por lo cual, cobra relevancia el reconocer a los sujetos sociales como portadores de una cultura social hecha de conocimientos, valores, actitudes y predisposiciones que no coinciden necesariamente con la cultura escolar y en especial con el currículo o programa que la institución se propone desarrollar ( Tenti, 2006 p. 27).

Es importante enunciarlos a partir de lo que ellos consideran que son, a partir de la horizontalidad asumida en la investigación realizada con los chicos de la escuela secundaria "Rey Netzahualcóyotl", ubicada en el municipio de Nezahualcóyotl; ellos antes de incluirse en una clasificación biológica o social, se reconocen desde sus modos de ser y estar, nombrándose como adolescentes-jóvenes desde lo que piensan y sienten que son dentro del espacio-tiempo que los ha constituido, sin la preocupación adultocéntrica de insertarse en una etapa lineal que determine su pensamiento y conducta, sino como un sujeto histórico "producido socialmente y que su autoconstitución pugna entre dos campos: instituido e instituyente" (Martínez, 2012, p.171).

En esa polifonía evocada en el espacio escolar, reflexionamos sobre las interacciones entre los diversos actores que socializaron a través de sus aprendizajes y experiencias en aulas y patios, como una autoeducación que enunciaría Dubet "en este sentido toda educación es una autoeducación, no es tan sólo una inculcación, es también un trabajo sobre sí mismo" (1998, p.14), adquiriendo así la secundaria otro sentido que visibilizó el poder y saber entre los autores, jóvenes y adultos al intercambiar microhistorias construidas en el mundo escolarizado en el cual 
estrecharon vínculos de identidad, por un lado con un mandato social, y por el otro en la reconstrucción de un mundo escolar donde aprenden a ser consigo mismos y con los otros, entonces la escuela también es:

Ese lugar donde se aprenden y olvidan cosas, donde se aprueban y suspenden exámenes, donde se difunden algunos saberes y donde se adquieren algunas destrezas y ciertas normas. Es ese lugar donde no sólo se enseña el conocimiento legítimo sino también el conocimiento esperado (la obediencia a la autoridad y el respeto a las reglas del juego) (...). Pero es también ese lugar donde suceden cosas divertidas, donde unos estudian lecciones y otros escriben en los cuadernos, donde habitan las ilusiones y en ocasionen también el desencanto, donde afloran las sonrisas aunque también emerge el llanto, donde se sufre con el dolor del fracaso y se goza con el placer del éxito, donde se dormita cuando sobreviene el hastío de las horas en la monotonía en las aulas y donde se escriben mensajes en los pupitres a golpe de bolígrafo o a punta de navaja ( Lomas, 2002, p.17).

Lo que acontece en la secundaria ha traspasado la estructura hegemónica que dio paso a su funcionamiento, hoy es necesario reconocer a la escuela secundaria de los adolescentes- jóvenes como un espacio donde interactúan subjetividades para formarse y transformarse. Por lo cual, cobra relevancia reconocer a los sujetos sociales como portadores de una cultura social hecha de conocimientos, valores, actitudes y predisposiciones que no coinciden necesariamente con la cultura escolar, y en especial con el currículo o programa propuesto a desarrollar por la institución (Tenti, 2006, p. 27).

Es por ello, que reconocer a los adolescentes-jóvenes como sujetos en proceso de formación, con poder ${ }^{6}$, saber y resistencia ${ }^{7}$ que reconstruye su propia subjetividad, posibilita mirar

\footnotetext{
${ }^{6}$ Definiendo el poder como un conjunto de acciones que tienen por propiedad influir sobre otras acciones; no es algo que se posea, sino que existe únicamente en ejercicio, pues se teje en las relaciones que esas acciones establecen entre sí. El poder, así entendido, revela un dinamismo que impide precisarlo sin tomar en consideración los aspectos que reviste y los factores que intervienen en su singularidad histórica; por ello, tampoco es algo fijo, ni una sucesión de estructuras (también fijas) que se relevan, sino una serie de multiplicidades inestables que se entrecruzan para dar forma a prácticas singulares de dominio y resistencia (Foucault, 1999)

${ }^{7}$ Una resistencia que no sólo se contrapone a la lógica social de la clase hegemónica sino que visibiliza la diversidad de conocimientos, ideologías y relaciones sociales en el aula.
}

Revista RedCA octubre 2020 - enero 2021 ISSN: 2594-2824 Vol. 3 Núm. 8 
a la secundaria desde lo estructural y lo subjetivos ambos referentes constitutivos de su proceso formativo.

Ellos acuden a la escuela secundaria no solo para dar cumplimiento al deber, sino para estar y ser con los otros, amigos(as), novio(a) y demás seres vivos. En esa convivencia juntos, inventan símbolos, códigos, emblemas y estéticas para adquirir entre sí una identidad que tiende a instaurar su propia alteridad (Reguillo, 2003, p.112), aquella que manifiesta una manera de vivir, ver y sentir que dista del orden social determinado, de ahí la relevancia de los acompañamientos y espacios.

Los encuentros que los jóvenes adolescentes establecen con sus pares en dicho espacio están ceñidos por una madeja múltiple de emociones gratas y no gratas que los acompañan en su proceso constitutivo, donde la comunicación es un elemento importante para las relaciones intersubjetivas allí presentes; así mismos los desencuentros traen consigo diferencias, tensiones, conflictos y violencias como parte constitutiva de su subjetividad juvenil.

Por lo tanto, las experiencias de los adolescentes-jóvenes hacen referencia a los vínculos diversos con seres apreciados, y que han sido significativos al grado de retenerlos en su memoria como "una experiencia que nos acaece, nos alcanza; que se apodera de nosotros, que nos tumba y nos transforma" (Larrosa en Contreras, 2010, p.102).

El adolescente-joven es un sujeto de la experiencia abierto a que algo lo conmueva y lo mueva en la creación de otros modos de ser, partiendo de esos momentos cotidianos sencillos que los hace sentirse vivos, el contacto con la naturaleza, una plática o reunión con su familia, o la relación con un maestro con el que aprendieron a bailar, cantar o a rebelarse, cada uno de estos acontecimientos deja huella en sus constituciones subjetivas de manera singular.

\section{A manera de cierre}

Caminar hacia el mismo lugar en momentos distintos de nuestra vida no nos da certeza, ni seguridad de haber llegado al punto específico, sino al contrario, ese momento de retorno es distinto cuando lo vislumbramos ya no como un punto de llegada sino como un punto de partida, y eso fue precisamente lo que me pasó en este proceso de investigación, al momento del cierre no concluyo con enunciaciones finales, sino con afirmaciones que nos dan apertura a continuar 
conociendo y reflexionando en esa necesidad de convenir con el otro, un andar por el mundo desde nuestros saberes, igualdades, diferencias y alteridades.

Algunos puntos de apertura expuestos en la presente discusión inacabada con respecto a los procesos de construcción subjetiva de los adolescentes-jóvenes en contextos de violencia son los siguientes:

-Las enunciaciones contextualizadas en orden social y económico dominante, connotan a una lectura mutirreferencial con respecto a la construcción subjetiva de los adolescentes-jóvenes de secundaria, articulándose así referentes epistémicos, teóricos, y metodológicos que dan cuenta de los procesos de construcción de los conocimientos conllevados en el trayecto de esta investigación; ya que en el proceso de construcción subjetiva con base al pensamiento potsabismal y el configuracionismo permitió reconocer que en la experiencia de los jóvenes se hacen presentes la estructuración, la subjetivación y la posibilidad de actuar frente a una realidad pensada desde esta interacción.

En las relaciones con los adolescentes-jóvenes podemos afirmar que la experiencia se relaciona a partir de las implicaciones como sujetos singulares al encontrarnos en un horizonte de igualdad frente a una situación problemática que nos hizo contarnos sobre asuntos que nos inquietaban y necesitábamos evocar para reconocernos como sujetos expuestos, sensibles, vulnerables, críticos y resistentes; inmersos en procesos configuradores de subjetividad a partir de lo que nos acontece, fragmenta y violenta.

Otro hallazgo que emerge de la presente investigación es entender la experiencia de los adolescentes-jóvenes desde sus diversas interacciones presentes en la secundaria como un escenario social de identidad, pertenencia y resistencia; ello como una posibilidad de nuevas investigaciones al ser los adolescentes-jóvenes una realidad en constante movimiento como protagonistas que marcan diferencias en los momentos históricos donde transitan, y por lo tanto la tarea del investigador no se remite a contemplar lo dado sino a continuar caminando para buscar los puntos de encuentro y desencuentro, como una apertura al encuentro consigo mismo y con los otros. 
La investigación abre otras interrogantes con respecto a los adolescentes-jóvenes, nos seduce a continuar andando en ese camino de formación que conlleva la experiencia de la investigación; una y otra vez nos encontraremos con esos hilos que comienzan a destejer historias

y al mismo tiempo a tejer otros modos de comprender el mundo con matices de sobrevivencia humana.

\section{Referencias bibliográficas}

Ardoino, J. (1988). Lo multirreferencial en torno a los problemas de investigación. Conferencia dictada el 21 de noviembre de 1988 en la UAM-Xochimilco.

Contreras, J. y Pérez N. (2010). La experiencia y la investigación educativa. España, Morata.

Corona, Sarah y Olaf K. (2012). El dialogo: metodologías horizontales en Ciencias Sociales en El dialogo: metodologías horizontales en Ciencias Sociales y Culturales. México: Gedisa.

De la Garza, E. (2018). La metodología configuracionista para la investigación. México: Universidad Autónoma Metropolitana Iztapalapa-Gedisa.

Dubet F. y Martuccelli D. (1998) En la escuela. España. Losada.

Foucault, M. (1991). Historia de la sexualidad I. La voluntad de saber. Madrid: Siglo XXI.

Foucault, M. (1999). Historia de la sexualidad II. El uso de los placeres. Madrid: Siglo XXI.

Larrosa J. (1995). Escuela, Poder y Subjetivación. Madrid. La piqueta.

Larrosa, J. (2006). Sobre la experiencia. En: Aloma: revista psicológica, ciencias de `educacio i de l'sport. Blanquerna. Núm.: 19. Barcelona.

Lomas, C. (2002). La vida en las aulas. Barcelona. Paidós.

Martínez, M. y Cubides, J. (2012). Acercamiento al uso de la categoría de "subjetividad política" en procesos investigativos en Claudia Piedrahita, Álvaro Díaz y Pablo Vommaro (Comps) Subjetividades politicas: desafíos y debates latinoamericanos. Bogotá: Universidad Distrital Francisco José de Caldas.p.p.177-176

Revista RedCA octubre 2020 - enero 2021 ISSN: 2594-2824 Vol. 3 Núm. 8 
Reguillo, R. (2003). Ciudadanías Juveniles en América Latina. En CIDPA Viña del Mar.

Santos, B. (2009). Una epistemología del sur. Buenos Aires, Argentina. Clacso-Siglo XXI.

Santos, B. (2010). Más allá del pensamiento abismal: de las líneas globales a una ecología de saberes. Para descolonizar occidente. Buenos Aires: CLACSO.

Tenti, E. (2006). Culturas juveniles y cultura escolar. La educación secundaria en América Latina, prioridad de la agenda 2000, Braslavsky. SEP

Touraine, A. (2000). ¿Podremos vivir juntos? México. Fondo de Cultura Económica. 